Invited Editorial:

\title{
Glial fibrillary acidic protein - a blood biomarker to differentiate neurodegenerative from psychiatric diseases
}

Henrik Zetterberg ${ }^{1,2,3,4,5^{*}}$

${ }^{1}$ Department of Psychiatry and Neurochemistry, Institute of Neuroscience and Physiology, the Sahlgrenska Academy at the University of Gothenburg, Mölndal, Sweden

${ }^{2}$ Clinical Neurochemistry Laboratory, Sahlgrenska University Hospital, Mölndal, Sweden

${ }^{3}$ Department of Neurodegenerative Disease, UCL Institute of Neurology, Queen Square,

London, $U K$

${ }^{4} U K$ Dementia Research Institute at UCL, London, UK

${ }^{5}$ Hong Kong Center for Neurodegenerative Diseases, Hong Kong, China

*Correspondence: henrik.zetterberg@clinchem.gu.se

Number of references: 4

Abstract count: 26 words

Word count (main text): 482 words

Blood glial fibrillary acidic protein (GFAP) concentration is higher in frontotemporal lobar degeneration (FTLD) than in primary psychiatric disorders (PPD) and predicts disease progression in FTLD.

Early symptoms of neurodegenerative and psychiatric diseases may be indistinguishable. It has become increasingly clear that the pathological changes underlying neurodegenerative diseases are well-established before clinical presentation. These pathological changes, previously only observable at autopsy, can now be detected using biomarkers (imaging- or fluid-based). ${ }^{1}$

To facilitate biomarker-based detection of neurodegeneration in clinical practice, easy-to-use blood tests for first-line testing would be of great value. Such tests have been developed for Alzheimer's disease pathology (plasma $A \beta 42 / A \beta 40$ ratio and phosphorylated tau species), 
and for neurofilament light (NfL), a more general biomarker for neuroaxonal injury in both acute and chronic neurological conditions. ${ }^{1}$ In contrast, it has been difficult to identify biomarkers for psychiatric diseases - none exists to date and the most general biomarker (NfL) is negative.

One protein that has emerged as a candidate blood biomarker for central nervous system (CNS) diseases is glial fibrillary acidic protein (GFAP). It is the major structural protein of the intermediate filaments in astrocytes, and its expression is strongly brain-enriched (https://www.proteinatlas.org/ENSG00000131095-GFAP). An enzyme-linked immunosorbent assay for cerebrospinal fluid (CSF) GFAP was developed more than 30 years ago, ${ }^{2}$ but it has struggled to find its place in clinical chemistry; group level differences across neurological conditions exist but not in a clinically meaningful manner (except for Alexander disease, a rare neurodegenerative disease caused by mutations in the GFAP gene, where CSF GFAP levels are sky high).

Recently, ultra-sensitive assays for GFAP have been developed, allowing for the reliable measurement of the protein in blood. Plasma or serum GFAP has been examined as a biomarker for stroke and traumatic brain injury, and differences have also been detected in neurodegenerative diseases. One surprising feature of this biomarker is that its blood concentration correlates better with CNS changes than its corresponding CSF concentration. ${ }^{3}$ This is potentially explained by direct release of GFAP into the bloodstream by astrocytic end-feet at the neurovascular unit.

Katisko et al. ${ }^{4}$ demonstrate that serum and whole blood GFAP concentrations are higher across a broad spectrum of FTLDs (no subtype-specific changes were observed) compared with both PPD and healthy controls. The results resemble recently obtained blood NfL data by the same research group and others and are important for several reasons:

- The two markers reflect different aspects of CNS disease; whilst NfL is a neuroaxonal injury marker, GFAP captures astrocytic components of the disease processes, a distinction that may be important in clinical trials (depending on the drug target).

- NfL is slow to change (weeks to months), whilst GFAP is fast (days), which may be relevant for disease monitoring.

- Astrocytic dysfunction is a proposed disease mechanism in PPD; the study by Katisko et al. ${ }^{4}$ suggest that, if this is the case, it is not detectable using blood GFAP. 
The results by Katisko et al. ${ }^{4}$ support implementation of blood GFAP in clinical laboratory practice. To facilitate such work, more studies on the influence of pre-analytical factors and other potential confounders are needed.

\section{Acknowledgements}

HZ is a Wallenberg Scholar supported by grants from the Swedish Research Council (\#201802532), the European Research Council (\#681712), Swedish State Support for Clinical Research (\#ALFGBG-720931), the Alzheimer Drug Discovery Foundation (ADDF), USA (\#201809-2016862), the AD Strategic Fund and the Alzheimer's Association (\#ADSF-21831376-C, \#ADSF-21-831381-C and \#ADSF-21-831377-C), the Olav Thon Foundation, the Erling-Persson Family Foundation, Stiftelsen för Gamla Tjänarinnor, Hjärnfonden, Sweden (\#FO2019-0228), the European Union's Horizon 2020 research and innovation programme under the Marie Skłodowska-Curie grant agreement No 860197 (MIRIADE), and the UK Dementia Research Institute at UCL.

\section{Conflicts of interest}

HZ has served at scientific advisory boards and/or as a consultant for Alector, Eisai, Denali, Roche Diagnostics, Wave, Samumed, Siemens Healthineers, Pinteon Therapeutics, Nervgen, AZTherapies, CogRx and Red Abbey Labs, has given lectures in symposia sponsored by Cellectricon, Fujirebio, Alzecure and Biogen, and is a co-founder of Brain Biomarker Solutions in Gothenburg AB (BBS), which is a part of the GU Ventures Incubator Program (outside submitted work).

\section{References}

1. Ehrenberg AJ, Khatun A, Coomans E, et al. Relevance of biomarkers across different neurodegenerative diseases. Alzheimers Res Ther 2020;12(1):56. doi: 10.1186/s13195020-00601-w [published Online First: 2020/05/15]

2. Rosengren LE, Ahlsen G, Belfrage M, et al. A sensitive ELISA for glial fibrillary acidic protein: application in CSF of children. J Neurosci Methods 1992;44(2-3):113-9. doi: 10.1016/0165-0270(92)90004-w [published Online First: 1992/09/01]

3. Pereira JB, Janelidze S, Smith R, et al. Plasma GFAP is an early marker of amyloid-beta but not tau pathology in Alzheimer's disease. Brain 2021 doi: 10.1093/brain/awab223 [published Online First: 2021/07/15]

4. Katisko K, Cajanus A, Huber N, et al. GFAP as a biomarker in frontotemporal dementia and primary psychiatric disorders: diagnostic and prognostic performance. J Neurol Neurosurg Psychiatry 2021 doi: 10.1136/jnnp-2021-326487 [published Online First: 2021/07/01] 